\title{
Effects of Magnetohydrodynamics and Heat Transfer in Casson Fluid Through a Channel
}

\author{
Wan Faezah Wan Azmi, Ahmad Qushairi Mohamad*, Yeak Su Hoe, Zaiton Mat Isa, \\ Sharidan Shafie
}

Department of Mathematical Sciences, Faculty of Science, Universiti Teknologi Malaysia, 81310 UTM Johor Bahru, Johor, Malaysia;

\begin{abstract}
Unsteady flow of Casson fluid past through a vertical channel has been studied by some researchers due to its importance of applications in science and technology. Therefore, the main purpose of this paper is to obtain exact solutions for unsteady free convection flows of Casson fluid with effects of magnetohydrodynamics (MHD) past through vertical channel. This paper is continued study from published article [18] with additional effects of magnetohydrodynamics (MHD). Dimensional governing equations are converted into dimensionless forms by using appropriate dimensionless variables. Dimensionless parameters are obtained through dimensionless process such as Casson fluid, time, Prandtl number, Grashof number and magnetic field. Laplace transform method is used to solve the dimensionless equations with associated initial and boundary conditions. Solutions for velocity and temperature profiles are obtained. Skin friction and Nusselt number are also calculated. The obtained analytical results for velocity and temperature are plotted graphically to discuss the influence of dimensionless parameters on profiles. It is observed that fluid velocity increases with increases of Grashof number, Gr and time, $t$ whereas it decreases with increases of Casson parameter, $\gamma$, magnetic field parameter, $M$ and Prandtl number, Pr. Besides that, it is found that temperature profiles decrease with high value of Prandtl number, Pr while increases with high value of time, $t$. In order to validate the results, the obtained results in limiting cases are compared with the published results and it is found to be in a mutual agreement.
\end{abstract}

Keywords: Casson fluid, Laplace transform, MHD, free convection flow.

*For correspondence: ahmadqushairi@utm.my

Received: 24 Nov 2020

Accepted: 15 August 2021

(C) Copyright Azmi. This article is distributed under the terms of the Creative Commons Attribution License, which permits unrestricted use and redistribution provided that the original author and source are credited.

\section{Introduction}

Free convection flow with the effect of MHD in Casson fluid had been continuedly studied due to its importance for the growth and development of living being. An example of literature which studied Casson fluid and solved numerically is done by Raju et al [1]. They analyzed the flow, heat and mass transfer behavior of Casson fluid past an exponentially permeable stretching surface with the presence of thermal radiation, magnetic field, viscous dissipation, heat source and chemical reaction. The results showed that Casson fluid has better performance of heat transfer compared to Newtonian fluid. Then, Tamoor et al [2] investigated the joule heating and MHD effects in dissipated stretched flow of Casson fluid. They implemented Homotopy Analysis Method (HAM) in this problem. The result was that the increase of Casson parameter and magnetic field can cause fluid velocity to diminish. Next, Nawaz, Naz and Awais [3] studied the effects of thermal conductivity on MHD axisymmetric free stream flow of Casson fluid. They used the same method as Tamoor et al. [2] to solve this problem. They found that Casson parameter increased and lead to the decrease in fluid velocity as well as increase of magnetic 
field which contributed to increase in fluid velocity. Besides that, Javed et al. [4] discussed Casson fluid flow due to torsional motion of cylinder with the effect of heat generation and absorption on axisymmetric. Built-in-Shooting method is been used to solve their problems numerically. They observed that the increment of Casson and magnetic parameter caused fluid velocity to decrease and the temperature profile to increase. Mabood and Das [5] focused on framing the features of melting heat transfer on MHD Casson fluid flow in a porous medium influenced by thermal radiation. They solved numerically by using Runge-Kutta Fehlberg-45 order method. They found the deceleration of fluid velocity due to the increase of Casson, magnetic field and permeability parameters. None of them solved problem analytically by using Laplace transform method.

Laplace transform method is one of the famous tools to solve problem analytically for Casson fluid flow on plates. Khalid et al.[6] investigated the unsteady Casson fluid flow with free convection and MHD effect through an oscillating vertical plate which is embedded in a porous medium. They found that the velocity decays in the presence of MHD, Casson parameter and porous medium. Then, Kataria and Patel [7] discussed heat \& mass transfer of Casson fluid with MHD effect. Its boundary conditions are oscillating vertical plate which is embedded in porous medium with ramped wall temperature. Kataria et al. [8] did further research on Casson fluid flow with the effects of chemical reaction, MHD and heat generation and absorption. It flows over an exponentially accelerated vertical plate which is embedded in porous medium with ramped wall temperature and surface concentration. Fluid motion tends to accelerate with the increase value of heat generation, decrease value of magnetic field, Casson parameter and chemical reaction parameter. Furthermore, Khan et al. [9] investigated similar problem as Kataria et al. [8] with additional effect of Newtonian heating which flows over a moving vertical plate embedded in a porous medium. Rao et al. [10] solved analytically problem of unsteady MHD Casson fluid flow in porous medium with heat and mass transfer. They solved fluid flow on exponentially accelerated vertical plate. Tassaddiq et al. [11] investigated Casson fluid flow with MHD, Newtonian heating, and porosity effect on the fixed vertical plate by using fractional model with Mittag-Leffler memory. Abdelhameed [12] studied unsteady free convection flow of Newtonian fluid with effect of magnetic field over an accelerated plate. Anwar [13] discussed the effect of thermal radiation and porosity on MHD Casson fluid flow past through a vertical plate with ramped wall velocity and temperature. They solved the problem by joining the Durbin method and Laplace transform. Then, Casson fluid flow in porous medium on Riga plate with thermal radiation and chemical reaction was studied by Bilal et al. [14]. They solved the fluid flow on an exponentially accelerated inclined vertical plate. None of them solved problem analytically for Casson fluid flow in channel by using Laplace transform.

Boundary conditions are important to determine the fluid motion behavior. There are many types of boundary conditions that had been studied. Literature on fluid flow in vertical channel by using Laplace transform technique to obtain exact solutions will be focused in this section. Earlier works done discussed Newtonian fluid with different effects such as Paul et al.[15] which discussed free convection effect with constant temperature and heat flux on walls. They observed that free convection has more effect in air compared to water. Then, Jha [16] investigated free convection flow on magnetic field in electrically conducting viscous fluid through a vertical channel. They showed that fluid velocity decreased with the increase of magnetic parameter. Besides that, Narahari [17] extended the problem as Paul et al. [15] with constant heat flux and radiation effects. They observed that velocity and temperature profiles decreased with the increase of radiation parameter. After that, Marneni [18] added mass transfer on fluid flow problem without constant heat flux and radiation effects as a new problem. Narahari et al. [19] studied exact solution for unsteady natural convection flow of viscous fluid with ramped wall temperature effect. Next, Jha et al.[20] presented free convective heat and mass transfer with diffusion thermo effect. The obtained result was that the Dufor effect enhanced the fluid temperature which means it can reduce time taken for fluid to reach steady state condition. Seth et al. [21] investigated Newtonian fluid in unsteady MHD free convection flow through a rotating vertical channel in porous medium with hall effects. Impulsive movement of one of the plates of the channel induced fluid to flow. Khan et al.[22] focused on the application of the fractal-fractional model of unsteady free convection Newtonian fluid flow in channel. None of them studied analytically for non-Newtonian fluid flow in channel. 
Recently, some researchers introduced new study on non-Newtonian fluid which is Casson fluid flow in vertical channel. Khan et al. [23] focused on thermal radiation effect on Casson fluid flow past through vertical channel. They solved problem analytically with fixed vertical plate and oscillating vertical plate. Ahmad Qushairi et al.[24] studied unsteady fee convection flow of Casson fluid past through a fixed channels. They solved problem by using Laplace transform method. Mallikarjuna et al. [25] solved numerically the problem of pulsatile Casson fluid flow in channel with MHD and slip velocity effects. Then, Bukhari et al. [26] extended the problem without slip velocity but with the additional effects of porosity and thermal radiation. They solved numerically by using finite difference approach method. Divya et al. [27] presented mathematical model of MHD Casson fluid flow in channel with heat and mass transfer effects. They solved the problems analytically by using perturbation method. Sheikh et al. [28] investigated Casson fluid flow in channel with applied external magnetic field. They solved the problem by using Laplace transform method.

To sum up, the literature that had been discussed as above stated that no study has been reported yet in investigating Casson fluid flow and heat transfer analysis past through vertical channel with MHD effects. Thus, the present work will focus on unsteady free convection flow of MHD Casson fluid in vertical channel. The analytical solutions are obtained by using the Laplace transform method. Laplace transform is one of the famous analytical techniques to solve problems in fluid mechanics which is involved with time dependent initial and boundary value problems. Unsteady state involves time dependent of initial and boundary value problems which is fitted with the proposed method to obtain exact solutions of governing equation.

\section{Problem Formulation}

Consider the unsteady free convection flow of incompressible Casson fluid past through a vertical channel which is separated by distance $d$ with constant temperature. The $x$-axis is in upward direction along the channel and $y$-axis is in normal direction to the channel. A uniform transverse magnetic field of strength $B_{0}$ is then applied parallel to $y$-axis. It is assumed that induced magnetic field, the external electric field and electric field due to polarization of charges are negligible. Initially at time $t^{*} \leq 0$, the fluid and channel are both at rest and assumed at the same temperature $T_{d}{ }^{*}$. Then, at the time $t^{*}>0$, plate temperature is raised to $T_{w}{ }^{*}$ at $y^{*}=0$ while plate temperature at $y^{*}=d$ is remain at constant temperature $T_{d}{ }^{*}$ and velocity for channel is $u^{*}(y, t)=0$. Then, under the usual Boussinesq's approximation, the corresponding partial differential equations for momentum and energy by Khalid et al. [6] are given as

$$
\begin{gathered}
\rho \frac{\partial u^{*}}{\partial t^{*}}=\mu\left(1+\frac{1}{\gamma}\right) \frac{\partial^{2} u^{*}}{\partial y^{* 2}}-\sigma B_{0}{ }^{2} u^{*}+\rho g \beta\left(T^{*}-T_{d}^{*}\right) \\
\rho c_{\mathrm{p}} \frac{\partial T^{*}}{\partial t^{*}}=k \frac{\partial^{2} T^{*}}{\partial y^{* 2}}
\end{gathered}
$$

with the associated initial and boundary conditions

$$
\begin{aligned}
& u^{*}\left(y^{*}, 0\right)=0, \quad T^{*}\left(y^{*}, 0\right)=T_{d}^{*} ; 0 \leq y^{*} \leq d, \\
& u^{*}\left(0, t^{*}\right)=0, \quad T^{*}\left(0, t^{*}\right)=T_{w}^{*} ; t^{*}>0, \\
& u^{*}\left(d, t^{*}\right)=0, \quad T^{*}\left(d, t^{*}\right)=T_{d}^{*} ; t^{*}>0 .
\end{aligned}
$$

where $u^{*}$ is the velocity component along $y$-axis, $\mu$ is the dynamic viscosity of fluid, $y$ is the non-Newtonian Casson parameter, $\sigma$ is the electrical conductivity, $\rho$ is the density of fluid, $g$ is the gravitational acceleration, $\beta$ is the heat transfer coefficient, $T$ is temperature of fluid, $c_{p}$ is the specific heat capacity of fluid at constant temperature, $k$ is thermal conductivity, $v$ is the kinematic viscosity of fluid. Introducing the following dimensionless variables: 


$$
u=\frac{u^{*} d}{v}, \quad y=\frac{y^{*}}{d}, t=\frac{t^{*} v}{d^{2}}, \quad T=\frac{T^{*}-T_{d}^{*}}{T_{w}^{*}-T_{d}^{*}}
$$

and substitute equation (4) into equations (1)-(3), obtained dimensionless partial differential equations as

$$
\begin{gathered}
\frac{\partial^{2} u}{\partial y^{2}}-\frac{1}{a}\left(\frac{\partial u}{\partial t}+M u\right)=-\frac{G r T}{a} \\
\frac{\partial^{2} T}{\partial y^{2}}-\operatorname{Pr} \frac{\partial T}{\partial t}=0
\end{gathered}
$$

with the associated initial and boundary conditions

$$
\begin{aligned}
& u(y, 0)=0, T(y, 0)=0 ; 0 \leq y \leq 1, \\
& u(0, t)=0, T(0, t)=1 ; t>0, \\
& u(1, t)=0, T(1, t)=0 ; t>0 .
\end{aligned}
$$

where $G r=\frac{d^{3} g \beta\left(T_{w}{ }^{*}-T_{d}{ }^{*}\right)}{v^{2}}$ is the Grashof number, $M=\frac{d^{2} \sigma B_{0}^{2}}{\mu}$ is the magnetic parameter, $\operatorname{Pr}=\frac{\mu c_{\mathrm{p}}}{k}$ is the Prandtl number and $a_{0}=1+\frac{1}{\gamma}, a=\frac{1}{a_{0}}$ are the constant parameters.

\section{Problem Solution}

\section{Temperature and Velocity Profiles}

In order to obtain the analytical solution of equations (5) and (6), apply Laplace transform into equations (5) and (6) subjected to the initial equations (7), yields

$$
\begin{gathered}
\frac{d^{2} \bar{u}(y, s)}{d y^{2}}-\left(\frac{s+M}{a}\right) \bar{u}(y, s)=-\frac{G r}{a} \bar{T}(y, s) \\
\frac{d^{2} \bar{T}(y, s)}{d y^{2}}-s \operatorname{Pr} \bar{T}(y, s)=0
\end{gathered}
$$

where $\bar{u}(y, s)$ is the Laplace velocity, $\bar{T}(y, s)$ is the Laplace temperature and $s$ is the transformation variable. The corresponding Laplace transform for boundary conditions are

$$
\begin{aligned}
& \bar{u}(0, s)=0, \bar{T}(0, s)=\frac{1}{s}, \\
& \bar{u}(1, s)=0, \bar{T}(1, s)=0 .
\end{aligned}
$$

The inverse Laplace transform of equations (8) and (9) by imposed of equation (10), give 


$$
\begin{aligned}
& u(y, t)=b_{5} \sum_{n=0}^{\infty}\left[u_{1}(y, t)\right]-b_{5} \sum_{n=0}^{\infty}\left[u_{2}(y, t)\right]-b_{5} \sum_{n=0}^{\infty}\left[u_{3}(y, t)\right]+b_{5} \sum_{n=0}^{\infty}\left[u_{4}(y, t)\right] \\
& -b_{5} \sum_{n=0}^{\infty}\left[u_{5}(y, t)\right]+b_{5} \sum_{n=0}^{\infty}\left[u_{6}(y, t)\right]+b_{5} \sum_{n=0}^{\infty}\left[u_{7}(y, t)\right]-b_{5} \sum_{n=0}^{\infty}\left[u_{8}(y, t)\right], \\
& T(y, t)=\sum_{n=0}^{\infty}\left[\operatorname{erfc}\left(\frac{(2 n+y) \sqrt{\operatorname{Pr}}}{2 \sqrt{t}}\right)-\operatorname{erfc}\left(\frac{(2 n+2-y) \sqrt{\operatorname{Pr}}}{2 \sqrt{t}}\right)\right] \text {, }
\end{aligned}
$$

where

$$
\begin{aligned}
& u_{1}(y, t)=\frac{1}{2} \exp \left(-b_{6} \sqrt{M}\right) \operatorname{erfc}\left(\frac{b_{6}}{2 \sqrt{t}}-\sqrt{M t}\right)+\frac{1}{2} \exp \left(b_{6} \sqrt{M}\right) \operatorname{erfc}\left(\frac{b_{6}}{2 \sqrt{t}}+\sqrt{M t}\right) \\
& u_{2}(y, t)=\frac{1}{2} \exp \left(-b_{7} \sqrt{M}\right) \operatorname{erfc}\left(\frac{b_{7}}{2 \sqrt{t}}-\sqrt{M t}\right)+\frac{1}{2} \exp \left(b_{7} \sqrt{M}\right) \operatorname{erfc}\left(\frac{b_{7}}{2 \sqrt{t}}+\sqrt{M t}\right) \text {, } \\
& u_{3}(y, t)=\operatorname{erfc}\left(\frac{b_{8}}{2 \sqrt{t}}\right) \\
& u_{4}(y, t)=\operatorname{erfc}\left(\frac{b_{9}}{2 \sqrt{t}}\right) \text {, } \\
& u_{5}(y, t)=\frac{1}{2} \exp \left(b_{4} t-b_{6} \sqrt{b_{4}+M}\right) \operatorname{erfc}\left(\frac{b_{6}}{2 \sqrt{t}}-\sqrt{\left(b_{4}+M\right) t}\right) \\
& +\frac{1}{2} \exp \left(b_{4} t+b_{6} \sqrt{b_{4}+M}\right) \operatorname{erfc}\left(\frac{b_{6}}{2 \sqrt{t}}+\sqrt{\left(b_{4}+M\right) t}\right) \\
& u_{6}(y, t)=\frac{1}{2} \exp \left(b_{4} t-b_{7} \sqrt{b_{4}+M}\right) \operatorname{erfc}\left(\frac{b_{7}}{2 \sqrt{t}}-\sqrt{\left(b_{4}+M\right) t}\right) \\
& +\frac{1}{2} \exp \left(b_{4} t+b_{7} \sqrt{b_{4}+M}\right) \operatorname{erfc}\left(\frac{b_{7}}{2 \sqrt{t}}+\sqrt{\left(b_{4}+M\right) t}\right), \\
& u_{7}(y, t)=\frac{1}{2} \exp \left(b_{4} t-b_{8} \sqrt{b_{4}}\right) \operatorname{erfc}\left(\frac{b_{8}}{2 \sqrt{t}}-\sqrt{b_{4} t}\right) \\
& +\frac{1}{2} \exp \left(b_{4} t+b_{8} \sqrt{b_{4}}\right) \operatorname{erfc}\left(\frac{b_{8}}{2 \sqrt{t}}+\sqrt{b_{4} t}\right), \\
& u_{8}(y, t)=\frac{1}{2} \exp \left(b_{4} t-b_{9} \sqrt{b_{4}}\right) \operatorname{erfc}\left(\frac{b_{9}}{2 \sqrt{t}}-\sqrt{b_{4} t}\right) \\
& +\frac{1}{2} \exp \left(b_{4} t+b_{9} \sqrt{b_{4}}\right) \operatorname{erfc}\left(\frac{b_{9}}{2 \sqrt{t}}+\sqrt{b_{4} t}\right) \text {, } \\
& \text { In which } b_{4}=\frac{M}{a \operatorname{Pr}-1}, b_{5}=\frac{G r}{M}, b_{6}(n)=(2+2 n-y) \sqrt{\frac{1}{a}}, b_{7}(n)=(2 n+y) \sqrt{\frac{1}{a}} \text {, }
\end{aligned}
$$


$b_{8}(n)=(2+2 n-y) \sqrt{\operatorname{Pr}}$, and $b_{9}(n)=(2 n+y) \sqrt{\operatorname{Pr}}$.

\section{Skin Friction and Nusselt number}

In the dimensionless form, the governing equation for skin friction and Nusselt number for Casson fluid is given as

$$
\begin{aligned}
& \tau=-\left.\left(1+\frac{1}{\gamma}\right) \frac{\partial u}{\partial y}\right|_{y=0}, \\
& N u=-\left[\frac{\partial T}{\partial y}\right]_{y=0} .
\end{aligned}
$$

Thus, skin friction of this problem can be expressed as

$$
\tau(t)=-\left(1+\frac{1}{\gamma}\right) b_{5} \sum_{n=0}^{\infty}\left[\begin{array}{c}
\tau_{u 1}(t)-\tau_{u 2}(t)-\tau_{u 3}(t)+\tau_{u 4}(t) \\
-\tau_{u 5}(t)+\tau_{u 6}(t)+\tau_{u 7}(t)-\tau_{u 8}(t)
\end{array}\right]
$$

where

$$
\begin{aligned}
\tau_{u 1}(t) & =\sum_{n=0}^{\infty}\left[\frac{\sqrt{M}}{2 \sqrt{a}} \operatorname{erfc}\left(\frac{1+n}{\sqrt{a t}}-\sqrt{M t}\right) \exp \left(-(2+2 n) \sqrt{\frac{M}{a}}\right)\right] \\
& +\sum_{n=0}^{\infty}\left[\frac{1}{2 \sqrt{\pi a t}} \exp \left(-(2+2 n) \sqrt{\frac{M}{a}}-\left(\frac{1+n}{\sqrt{a t}}-\sqrt{M t}\right)^{2}\right)\right] \\
& -\sum_{n=0}^{\infty}\left[\frac{\sqrt{M}}{2 \sqrt{a}} \operatorname{erfc}\left(\frac{1+n}{\sqrt{a t}}+\sqrt{M t}\right) \exp \left((2+2 n) \sqrt{\frac{M}{a}}\right)\right] \\
& +\sum_{n=0}^{\infty}\left[\frac{1}{2 \sqrt{\pi a t}} \exp \left((2+2 n) \sqrt{\frac{M}{a}}-\left(\frac{1+n}{\sqrt{a t}}+\sqrt{M t}\right)^{2}\right)\right]
\end{aligned}
$$




$$
\begin{aligned}
& \tau_{u 2}(t)=-\sum_{n=0}^{\infty}\left[\frac{\sqrt{M}}{2 \sqrt{a}} \operatorname{erfc}\left(\frac{n}{\sqrt{a t}}-\sqrt{M t}\right) \exp \left(-2 n \sqrt{\frac{M}{a}}\right)\right] \\
& -\sum_{n=0}^{\infty}\left[\frac{1}{2 \sqrt{\pi a t}} \exp \left(-2 n \sqrt{\frac{M}{a}}-\left(\frac{n}{\sqrt{a t}}-\sqrt{M t}\right)^{2}\right)\right] \\
& +\sum_{n=0}^{\infty}\left[\frac{\sqrt{M}}{2 \sqrt{a}} \operatorname{erfc}\left(\frac{n}{\sqrt{a t}}+\sqrt{M t}\right) \exp \left(2 n \sqrt{\frac{M}{a}}\right)\right] \\
& -\sum_{n=0}^{\infty}\left[\frac{1}{2 \sqrt{\pi a t}} \exp \left(2 n \sqrt{\frac{M}{a}}-\left(\frac{n}{\sqrt{a t}}+\sqrt{M t}\right)^{2}\right)\right] \text {, } \\
& \tau_{u 3}(t)=\sum_{n=0}^{\infty}\left[\sqrt{\frac{P r}{\pi t}} \exp \left(-\frac{\operatorname{Pr}(n+1)^{2}}{t}\right)\right] \\
& \tau_{u 4}(y, t)=-\sum_{n=0}^{\infty}\left[\sqrt{\frac{P r}{\pi t}} \exp \left(-\frac{P r n^{2}}{t}\right)\right] \\
& \tau_{u 5}(t)=\sum_{n=0}^{\infty}\left[\frac{\sqrt{b_{4}+M}}{2 \sqrt{a}} \operatorname{erfc}\left(\frac{1+n}{\sqrt{a t}}-\sqrt{\left(b_{4}+M\right) t}\right) \exp \left(b_{4} t-(2+2 n) \sqrt{\frac{b_{4}+M}{a}}\right)\right] \\
& +\sum_{n=0}^{\infty}\left[\frac{1}{2 \sqrt{\pi a t}} \exp \left(b_{4} t-(2+2 n) \sqrt{\frac{b_{4}+M}{a}}-\left(\frac{1+n}{\sqrt{a t}}-\sqrt{\left(b_{4}+M\right) t}\right)^{2}\right)\right] \\
& -\sum_{n=0}^{\infty}\left[\frac{\sqrt{b_{4}+M}}{2 \sqrt{a}} \operatorname{erfc}\left(\frac{1+n}{\sqrt{a t}}+\sqrt{\left(b_{4}+M\right) t}\right) \exp \left(b_{4} t+(2+2 n) \sqrt{\frac{b_{4}+M}{a}}\right)\right] \\
& +\sum_{n=0}^{\infty}\left[\frac{1}{2 \sqrt{\pi a t}} \exp \left(b_{4} t+(2+2 n) \sqrt{\frac{b_{4}+M}{a}}-\left(\frac{1+n}{\sqrt{a t}}+\sqrt{\left(b_{4}+M\right) t}\right)^{2}\right)\right] \text {, } \\
& \tau_{u 6}(t)=-\sum_{n=0}^{\infty}\left[\frac{\sqrt{b_{4}+M}}{2 \sqrt{a}} \operatorname{erfc}\left(\frac{n}{\sqrt{a t}}-\sqrt{\left(b_{4}+M\right) t}\right) \exp \left(b_{4} t-2 n \sqrt{\frac{b_{4}+M}{a}}\right)\right] \\
& -\sum_{n=0}^{\infty}\left[\frac{1}{2 \sqrt{\pi a t}} \exp \left(b_{4} t-2 n \sqrt{\frac{b_{4}+M}{a}}-\left(\frac{n}{\sqrt{a t}}-\sqrt{\left(b_{4}+M\right) t}\right)^{2}\right)\right] \\
& +\sum_{n=0}^{\infty}\left[\frac{\sqrt{b_{4}+M}}{2 \sqrt{a}} \operatorname{erfc}\left(\frac{n}{\sqrt{a t}}+\sqrt{\left(b_{4}+M\right) t}\right) \exp \left(b_{4} t+2 n \sqrt{\frac{b_{4}+M}{a}}\right)\right] \\
& -\sum_{n=0}^{\infty}\left[\frac{1}{2 \sqrt{\pi a t}} \exp \left(b_{4} t+2 n \sqrt{\frac{b_{4}+M}{a}}-\left(\frac{n}{\sqrt{a t}}+\sqrt{\left(b_{4}+M\right) t}\right)^{2}\right)\right]
\end{aligned}
$$




$$
\begin{aligned}
& \tau_{u 7}(t)=\sum_{n=0}^{\infty}\left[\frac{\sqrt{P r b_{4}}}{2} \operatorname{erfc}\left((1+n) \sqrt{\frac{P r}{t}}-\sqrt{b_{4} t}\right) \exp \left(b_{4} t-(2+2 n) \sqrt{P r b_{4}}\right)\right] \\
& +\sum_{n=0}^{\infty}\left[\frac{1}{2} \sqrt{\frac{P r}{\pi t}} \exp \left(b_{4} t-(2+2 n) \sqrt{P r b_{4}}-\left((1+n) \sqrt{\frac{P r}{t}}-\sqrt{b_{4} t}\right)^{2}\right)\right] \\
& -\sum_{n=0}^{\infty}\left[\frac{\sqrt{P r b_{4}}}{2} \operatorname{erfc}\left((1+n) \sqrt{\frac{P r}{t}}+\sqrt{b_{4} t}\right) \exp \left(b_{4} t+(2+2 n) \sqrt{\operatorname{Prb}_{4}}\right)\right] \\
& +\sum_{n=0}^{\infty}\left[\frac{1}{2} \sqrt{\frac{P r}{\pi t}} \exp \left(b_{4} t+(2+2 n) \sqrt{P r b_{4}}-\left((1+n) \sqrt{\frac{P r}{t}}+\sqrt{b_{4} t}\right)^{2}\right)\right], \\
& \tau_{u 8}(t)=-\sum_{n=0}^{\infty}\left[\frac{1}{2} \sqrt{\operatorname{Prb}_{4}} \operatorname{erfc}\left(n \sqrt{\frac{P r}{t}}-\sqrt{b_{4} t}\right) \exp \left(b_{4} t-2 n \sqrt{\operatorname{Prb}_{4}}\right)\right] \\
& -\sum_{n=0}^{\infty}\left[\frac{1}{2} \sqrt{\frac{P r}{\pi t}} \exp \left(b_{4} t-2 n \sqrt{P r b_{4}}-\left(n \sqrt{\frac{P r}{t}}-\sqrt{b_{4} t}\right)^{2}\right)\right] \\
& +\sum_{n=0}^{\infty}\left[\frac{1}{2} \sqrt{P r b_{4}} \operatorname{erfc}\left(n \sqrt{\frac{P r}{t}}+\sqrt{b_{4} t}\right) \exp \left(b_{4} t+2 n \sqrt{P r b_{4}}\right)\right] \\
& -\sum_{n=0}^{\infty}\left[\frac{1}{2} \sqrt{\frac{P r}{\pi t}} \exp \left(b_{4} t+2 n \sqrt{P r b_{4}}-\left(n \sqrt{\frac{P r}{t}}+\sqrt{b_{4} t}\right)^{2}\right)\right] \text {. }
\end{aligned}
$$

And Nusselt number can be expressed as

$$
N u=\sum_{n=0}^{\infty}\left[\sqrt{\frac{P r}{\pi t}} \exp \left(-n^{2} \frac{\operatorname{Pr}}{t}\right)-\sqrt{\frac{P r}{\pi t}} \exp \left(-(n+1)^{2} \frac{\operatorname{Pr}}{t}\right)\right]
$$

\section{Results and discussion}

Limiting case has been considered in this problem in order to check solutions accuracy. Validation process has been done by comparing solution with Ahmad Qushairi et al. [24] in which no MHD effect is considered in unsteady free convection of Casson fluid flow. This can be done by allowing $M$ is equal to zero in equation (11). It is found that the identical results are obtained as shown in Figure 1. Hence, the solution accuracy of this problem is confirmed. 


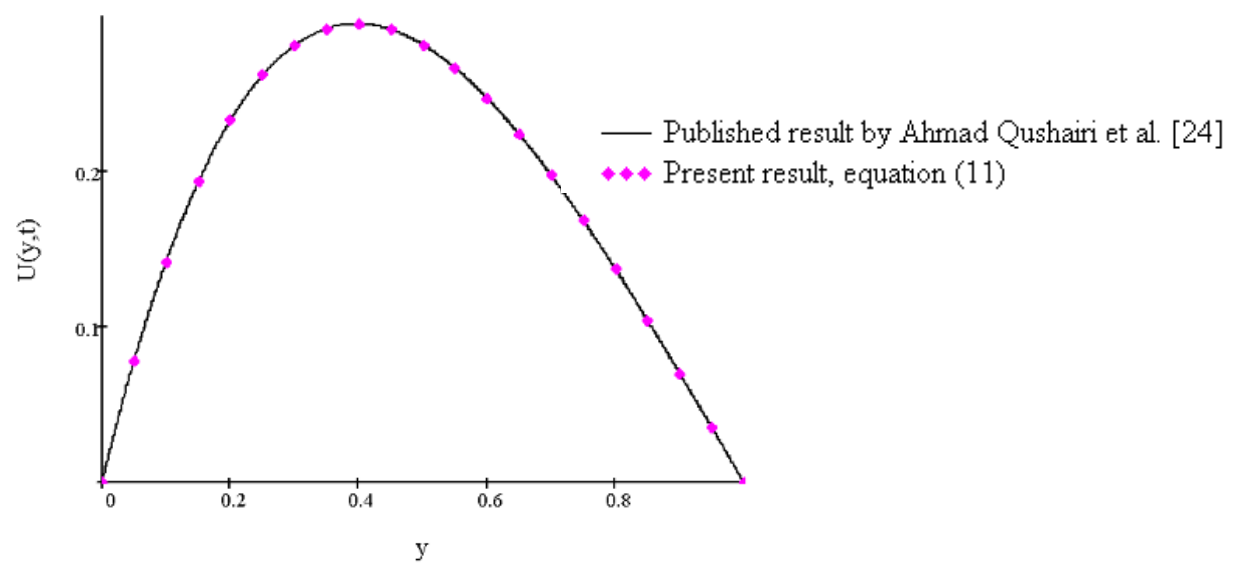

Figure 1. Comparison of velocity profile $u(y, t)$ from equation (11) with Ahmad Qushairi et al. [24].

Figure 2 displays velocity profiles for various values of Casson parameters $\gamma$. From the observation, fluid velocity decreases as Casson parameter increases due to high viscosity of fluid. Casson parameter is not applied as fluid moves towards center between bounding surface. Further observation from this figure, large value of Casson parameter results to Newtonian fluid behavior. Therefore, its velocity boundary layer thickness is larger than those for Newtonian fluid due to plasticity of Casson fluid. Decreasing of Casson parameter due to increasing in plasticity leads to increment in velocity boundary layer thickness due to increment in momentum boundary layer. The influence of magnetic field $M$ on velocity profiles is exhibited in Figure 3 . It is observed that the velocity decreases with increasing of magnetic field. Electric current is generated in fluid when magnetic field is applied to moving electrically conducting fluid. Interaction between induced currents and magnetic field produce a resistive force known as Lorentz force which is an external force works in the opposite direction of the fluid which retards the fluid flow. It is similar with the drag force. Furthermore, increment in magnetic parameter $M$ leads to increment of resistive forces which resist the fluid flow, thus fluid velocity is reduced. Figure 4 illustrates velocity profiles with various thermal Grashof number Gr. Thermal Grashof number is defined as the ratio of thermal buoyancy force to viscous hydrodynamic force (Kataria et al. [7]). The thermal buoyancy force is dominant during free convection process which results to increment in Grashof number Gr. Therefore, density of fluid decreases and small viscous effects in momentum equation which leads to increment in fluid velocity. As referred to Saqib et al. [29], buoyancy force is opposing fluid flow when value of $\mathrm{Gr}$ is negative whereas buoyancy force is supporting fluid flow when value of $\mathrm{Gr}$ is positive. Figure 5 and Figure 7 depict velocity and temperature profiles with different values of Prandtl number, Pr. Prandtl number is described as the ratio of kinematic viscosity to thermal diffusivity as referred to Das et al. [30]. Increases Prandtl number in the fluid flow will reduce thermal conductivity and increase fluid viscosity. Consequently, the fluid becomes thick and increase in viscous force which results to decrease in fluid velocity. The low rate of thermal diffusion causes arising in velocity boundary layer thickness. Besides that, the thermal boundary layer thickness decreases since decreasing fluid thermal conductivity with increasing Prandtl number which leads to decrease in temperature profiles. Prandtl number influences thickness of momentum and thermal boundary layers in heat transfer problems. Figure 6 and 8 show the behavior of velocity and temperature profiles with the time changes. It is found that velocity and temperature increase when the value of time $t$ is increased. It is due to the external energy that supply to the fluid flow which results in increasing fluid particle movement when time is increasing. Its results in increasing of fluid velocity and temperature. 


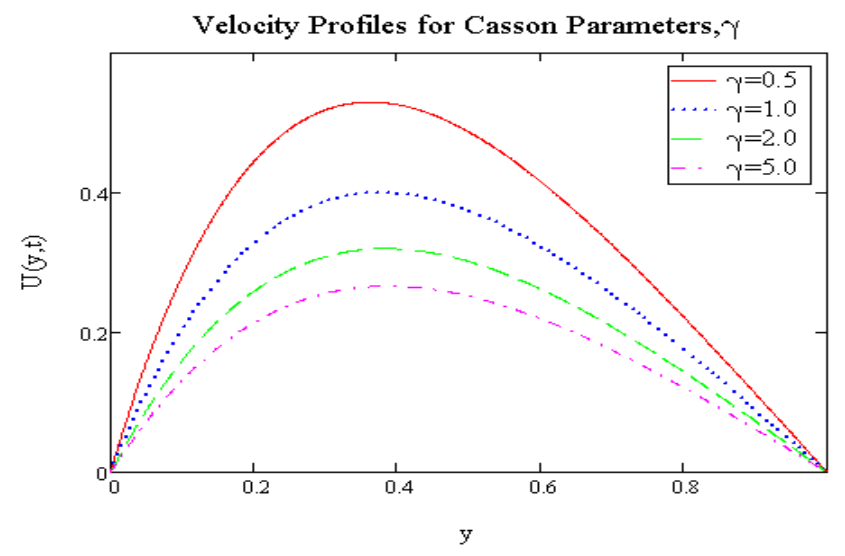

Figure 2. Velocity profiles for different values of Casson parameters with $M=1.0, \operatorname{Pr}=6.0, \mathrm{Gr}=5.0$ and $t=1.0$.

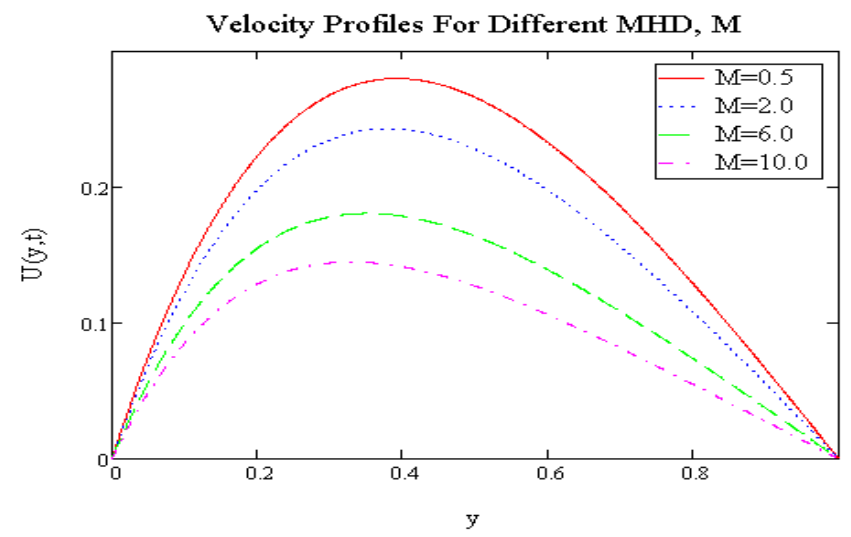

Figure 3. Velocity profiles for different values of Magnetic field parameters. with $M=1.0, \operatorname{Pr}=6.0, G r=5.0$ and $t=1.0$.

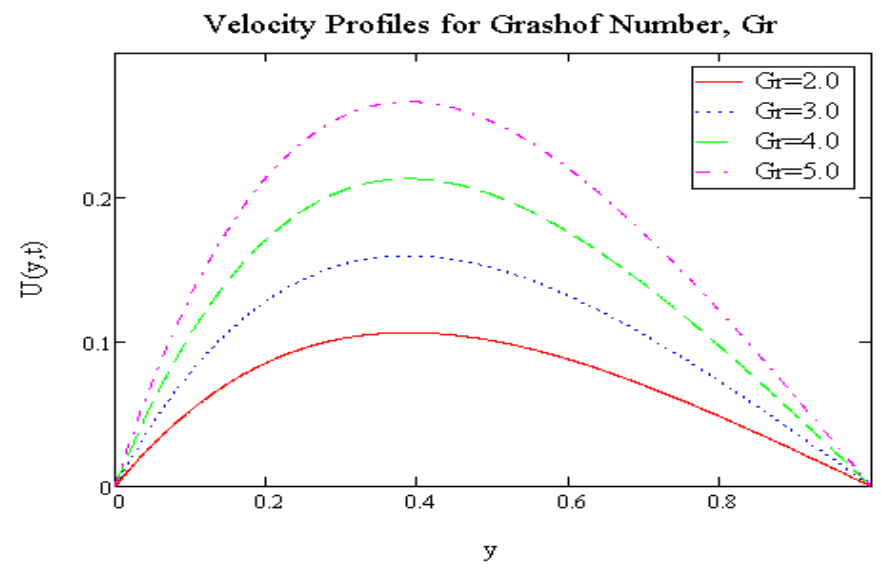

Figure 4. Velocity profiles for different values of Grashof numbers. with $M=1.0, \operatorname{Pr}=6.0, G r=5.0$ and $t=1.0$. 


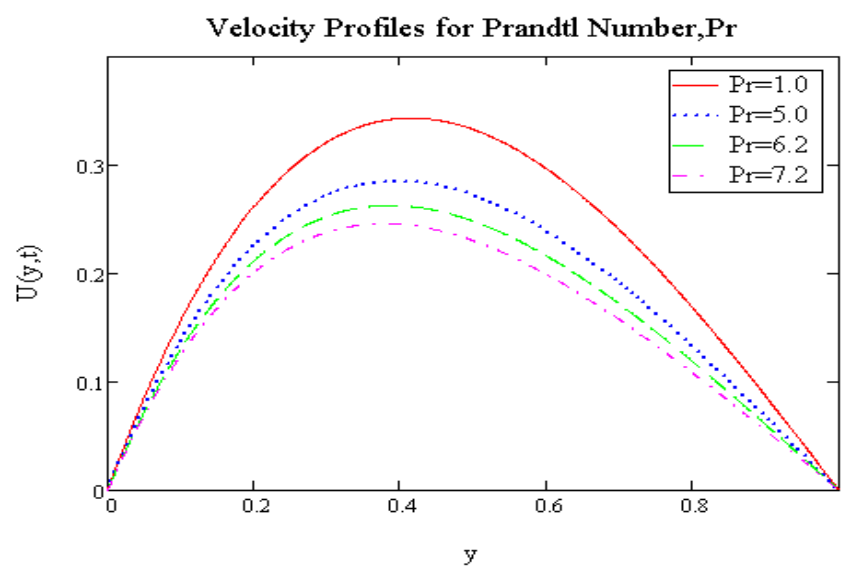

Figure 5. Velocity profiles for different values of Prandtl numbers. with $M=1.0, \operatorname{Pr}=6.0, G r=5.0$ and $t=1.0$.

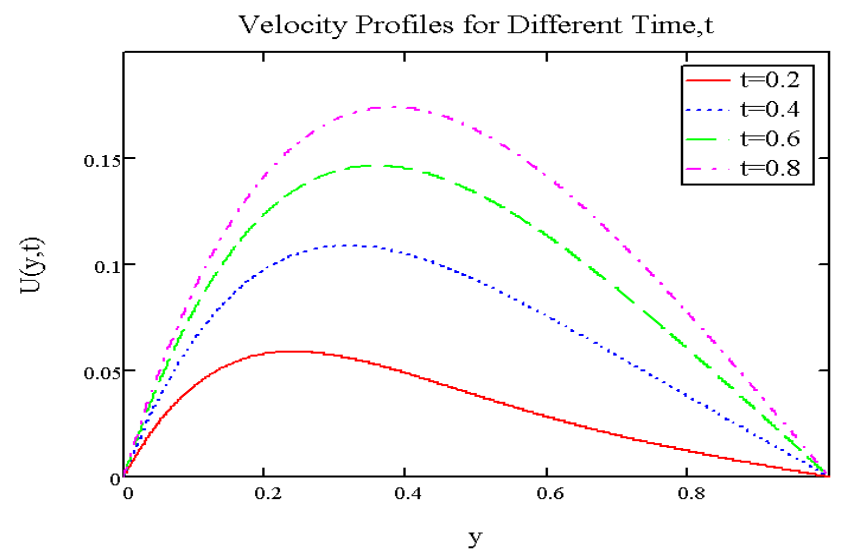

Figure 6. Velocity profiles for different values of time with $M=1.0, \operatorname{Pr}=6.0, G r=5.0$ and $t=1.0$.

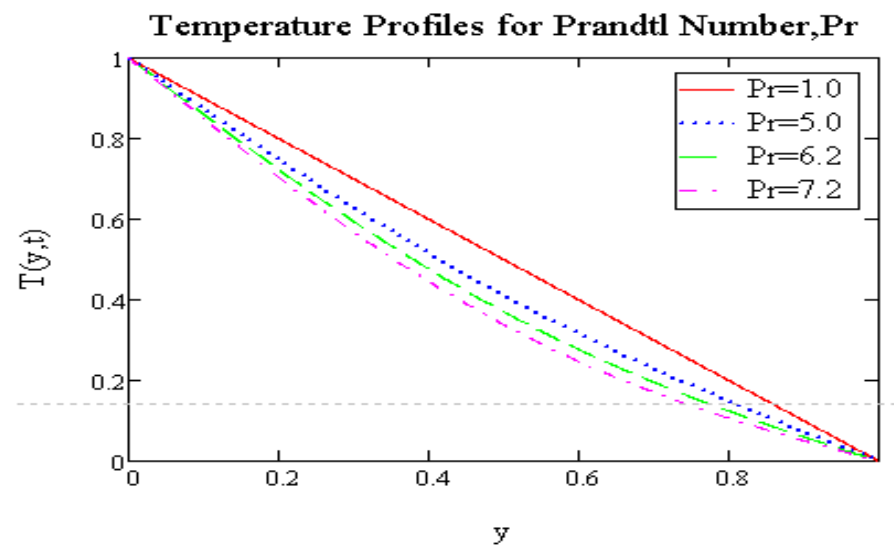

Figure 7. Temperature profiles for different values of Prandtl numbers. with $M=1.0, \operatorname{Pr}=6.0, G r=5.0$ and $t=1.0$. 


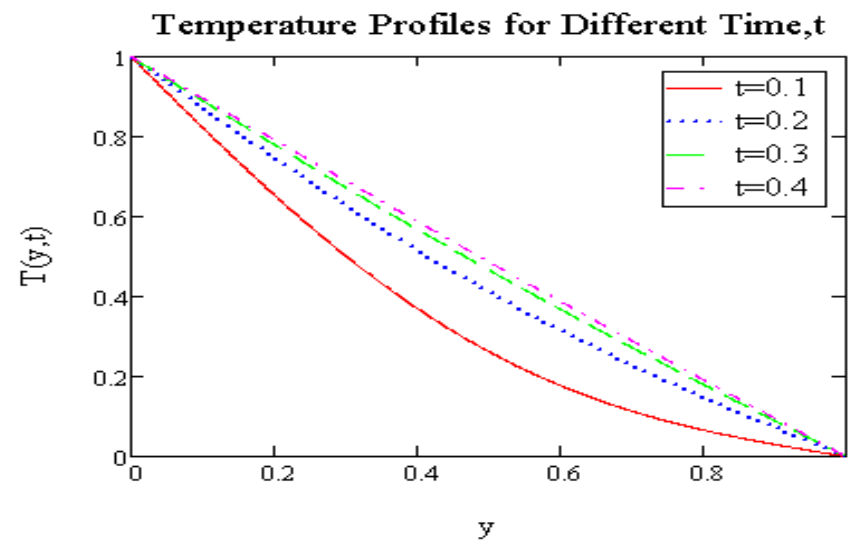

Figure 8. Temperature profiles for different values of time. with $M=1.0, \operatorname{Pr}=6.0, G r=5.0$ and $t=1.0$.

Table 1 shows the variation of skin friction for different values of related parameters. Skin friction is increasing due to increase in Casson parameter, Magnetic field and Prandtl number whereas decrease in time and Grashof number. Skin friction with negative value means that drag force is applied to the fluid due to the plate. Table 2 indicates Nusselt number with variations of parameters such as Prandtl number Pr and time $t$. It can be observed that, arising in Nusselt number due to increasing in Prandtl number and decreasing with time. Nusselt number can indicates either heat transfer by convection or conduction is dominant.

Table 1. Variation of skin friction, $t$ for different parameters.

\begin{tabular}{cccccc}
\hline$t$ & $P r$ & $M$ & $G r$ & $\gamma$ & $\tau$ \\
\hline $\mathbf{0 . 2 0}$ & $\mathbf{5 . 0 0}$ & $\mathbf{0 . 5 0}$ & $\mathbf{2 . 0 0}$ & $\mathbf{0 . 5 0}$ & -2.256 \\
$\mathbf{0 . 5 0}$ & 5.00 & 0.50 & 2.00 & 0.50 & -3.490 \\
$\mathbf{0 . 8 0}$ & 5.00 & 0.50 & 2.00 & 0.50 & -4.275 \\
0.20 & $\mathbf{6 . 2 0}$ & 0.50 & 2.00 & 0.50 & -2.123 \\
0.20 & $\mathbf{7 . 2 0}$ & 0.50 & 2.00 & 0.50 & -2.031 \\
0.20 & 5.00 & $\mathbf{1 . 0 0}$ & 2.00 & 0.50 & -2.225 \\
0.20 & 5.00 & $\mathbf{2 . 0 0}$ & 2.00 & 0.50 & -2.165 \\
0.20 & 5.00 & 0.50 & $\mathbf{3 . 0 0}$ & 0.50 & -3.385 \\
0.20 & 5.00 & 0.50 & $\mathbf{5 . 0 0}$ & 0.50 & -5.641 \\
0.20 & 5.00 & 0.50 & 2.00 & $\mathbf{1 . 0 0}$ & -1.092 \\
0.20 & 5.00 & 0.50 & 2.00 & $\mathbf{2 . 0 0}$ & -0.648 \\
\hline
\end{tabular}

Table 2. Variation of Nusselt, Nu number for different parameters.

\begin{tabular}{ccc}
\hline$t$ & $\operatorname{Pr}$ & $N u$ \\
\hline $\mathbf{0 . 1}$ & $\mathbf{5 . 0}$ & 3.989 \\
$\mathbf{0 . 2}$ & 5.0 & 2.821 \\
$\mathbf{0 . 3}$ & 5.0 & 2.303 \\
0.1 & 6.2 & 4.442 \\
0.1 & 7.2 & 4.787 \\
\hline
\end{tabular}




\section{Conclusions}

Analytical solutions for unsteady free convection flow of MHD Casson fluid through a vertical channel has been discussed in this thesis. The Laplace and inverse Laplace transform method had been used to attain analytical solutions for this problem. Finally, the solutions are satisfactory with related initial and boundary conditions. The obtained solutions are discussed graphically with the effects of Casson parameter $\gamma$, Grashof number Gr, Prandtl number Pr, time parameter $t$ and Magnetic parameter $M$. The velocity and temperature profiles increase when $t$ and $G r$ increase but decrease when $\operatorname{Pr}, \gamma$ and $M$ increase. skin friction increases with the increase of Casson parameter, $\gamma$, magnetic field parameter, $M$ and Prandtl number, Pr while decreases with Grashof number, Gr and time, $t$. Besides that, Nusselt number, $\mathrm{Nu}$ increases with high value of Prandtl number, $\mathrm{Pr}$ and decreases with high values of time, $t$. Solution (11) is found in excellent agreement with those obtained by Qushairi [18].

\section{Conflicts of interest}

The authors declare that there is no conflict of interest regarding the publication of this paper.

\section{Funding statement}

The authors would like to acknowledge the Ministry of Higher Education Malaysia and Research Management Centre-UTM, University Technology Malaysia (UTM) for financial support through vote numbers [17J98, FRGS/1/2019/STG06/UTM/02/22 and 08G33, 07G70, 07G72, 07G76, 07G77].

\section{References}

[1] C. S. K. Raju, N. Sandeep, V. Sugunamma, M. J. Babu, and J. V. R. Reddy, "Engineering Science and Technology, Heat and mass transfer in magnetohydrodynamic Casson fluid over an exponentially permeable stretching surface," Eng. Sci. Technol. an Int. J., vol. 19, no. 1, pp. 45-52, 2016.

[2] M. Tamoor, M. Waqas, M. I. Khan, A. Alsaedi, and T. Hayat, "Results in Physics Magnetohydrodynamic flow of Casson fluid over a stretching cylinder," Results Phys., vol. 7, pp. 498-502, 2017.

[3] M. Nawaz, R. Naz, and M. Awais, "Magnetohydrodynamic axisymmetric flow of Casson fluid with variable thermal conductivity and free stream," Alexandria Eng. J., vol. 57, no. 3, pp. 2043-2050, 2018.

[4] M. F. Javed, M. I. Khan, N. B. Khan, and R. Muhammad, "Axisymmetric fl ow of Casson fl uid by a swirling cylinder," Results Phys., vol. 9, no. March, pp. 1250-1255, 2018.

[5] F. Mabood and K. Das, "Outlining the impact of melting on MHD Casson fl uid fl ow past a stretching sheet in a porous medium with radiation," Heliyon, no. December 2018, p. e01216, 2019.

[6] A. Khalid, I. Khan, A. Khan, and S. Shafie, "Unsteady MHD free convection flow of Casson fluid past over an oscillating vertical plate embedded in a porous medium," Eng. Sci. Technol. an Int. J., vol. 18, no. 3, pp. 309-317, 2015.

[7] H. R. Kataria and H. R. Patel, "Heat and mass transfer in magnetohydrodynamic (MHD) Casson fluid flow past over an oscillating vertical plate embedded in porous medium with ramped wall temperature," Propuls. Power Res., vol. 7, no. 3, pp. 257-267, 2018.

[8] H. R. Kataria and H. R. Patel, "Effects of chemical reaction and heat generation/absorption on magnetohydrodynamic (MHD) Casson fluid flow over an exponentially accelerated vertical plate embedded in porous medium with ramped wall temperature and ramped surface concentration," Propuls. Power Res., no. 2019, 2019.

[9] D. Khan, A. Khan, I. Khan, F. Ali, F. ul Karim, and I. Tlili, "Effects of Relative Magnetic Field, Chemical Reaction, Heat Generation and Newtonian Heating on Convection Flow of Casson Fluid over a Moving Vertical Plate Embedded in a Porous Medium," Sci. Rep., vol. 9, no. 1, pp. 1-18, 2019.

[10] S. Ramalingeswara Rao, G. Vidyasagar, and G. V. S. R. Deekshitulu, "Unsteady MHD free convection Casson fluid flow past an exponentially accelerated infinite vertical porous plate through porous medium in the presence of radiation absorption with heat generation/absorption," Mater. Today Proc., vol. 42, pp. 1608-1616, 2019.

[11] A. Tassaddiq, I. Khan, K. Sooppy Nisar, and J. Singh, "MHD flow of a generalized Casson fluid with 
Newtonian heating: A fractional model with Mittag-Leffler memory," Alexandria Eng. J., vol. 59, no. 5, pp. 3049-3059, 2020.

[12] T. N. Abdelhameed, "Entropy generation analysis for MHD flow of water past an accelerated plate," Sci. Rep., vol. 11, no. 1, pp. 1-11, 2021.

[13] T. Anwar, P. Kumam, and W. Watthayu, "Unsteady MHD natural convection flow of Casson fluid incorporating thermal radiative flux and heat injection/suction mechanism under variable wall conditions," Sci. Rep., vol. 11, no. 1, pp. 1-15, 2021.

[14] S. Bilal, K. K. Asogwa, H. Alotaibi, M. Y. Malik, and I. Khan, "Analytical treatment of radiative Casson fluid over an isothermal inclined Riga surface with aspects of chemically reactive species," Alexandria Eng. J., vol. 60, no. 5, pp. 4243-4253, 2021.

[15] T. Paul, B. K. Jha, and A. K. Singh, "Transient free convective flow in a vertical channel with constant temperature and constant heat flux on walls," Heat Mass Transf. und Stoffuebertragung, vol. 32, no. 1-2, pp. 61-63, 1996.

[16] B. K. Jha, "Effects of Applied Magnetic Field on Transient Free-Convective Flow in a Vertical Channel," Indian J. pure Appl. Math., vol. 29, pp. 441-445, 1998.

[17] M. Narahari, "Natural Convection in Unsteady Couette Flow Between Two Vertical Parallel Plates in the Presence of Constant Heat Flux and Radiation," Appl. Sci., pp. 73-78, 2008.

[18] N. Marneni, "Transient Free Convection Flow Between Two Long Vertical Parallel Plates with Constant Temperature and Mass Diffusion," vol. II, 2008.

[19] M. Narahari and V. R. Raghavan, "Natural Convection Flow in Vertical Channel Due To Ramped Wall Temperature at One Boundary," pp. 1-8, 2009.

[20] B. K. Jha and A. O. Ajibade, "Diffusion-Thermo Effects on Free Convective Heat and Mass Transfer Flow in a Vertical Channel With Symmetric Boundary Conditions," J. Heat Transfer, vol. 133, no. 5, p. 052502, 2011.

[21] G. S. Seth, B. Kumbhakar, and R. Sharma, "Unsteady hydromagnetic natural convection flow of a heat absorbing fluid within a rotating vertical channel in porous medium with hall effects," J. Appl. Fluid Mech., vol. 8, no. 4, pp. 767-779, 2015.

[22] D. Khan, G. Ali, A. Khan, I. Khan, Y. M. Chu, and K. S. Nisar, "A new idea of fractal-fractional derivative with power law kernel for free convection heat transfer in a channel flow between two static upright parallel plates," Comput. Mater. Contin., vol. 65, no. 2, pp. 1237-1251, 2020.

[23] I. Khan, M. Saqib, and F. Ali, "Application of time-fractional derivatives with non-singular kernel to the generalized convective flow of Casson fluid in a microchannel with constant walls temperature," Eur. Phys. J. Spec. Top., vol. 226, no. 16-18, pp. 3791-3802, 2017.

[24] A. Q. Mohamad, J. Lim Yeou, S. Shafie, I. Khan, and Z. Ismail, "Exact solution for unsteady free convection flow of Casson fluid in vertical channel," MATEC Web Conf., vol. 189, p. 01007, 2018.

[25] B. Mallikarjuna, S. Ramprasad, and Y. S. K. Chakravarthy, "Multiple Slip and Inspiration Effects on Hydromagnetic Casson Fluid in a Channel with Stretchable Walls," Int. J. Heat Technol., vol. 38, no. 4, pp. 817-826, 2020.

[26] Z. Bukhari, A. Ali, Z. Abbas, and H. Farooq, "The pulsatile flow of thermally developed nonNewtonian Casson fluid in a channel with constricted walls," AIP Adv., vol. 11, no. 2, 2021.

[27] B. B. Divya, G. Manjunatha, C. Rajashekhar, H. Vaidya, and K. V. Prasad, "Analysis of temperature dependent properties of a peristaltic MHD flow in a non-uniform channel: A Casson fluid model," Ain Shams Eng. J., vol. 12, no. 2, pp. 2181-2191, 2021.

[28] N. A. Sheikh, D. L. C. Ching, T. Abdeljawad, I. Khan, M. Jamil, and K. S. Nisar, "A fractal-fractional model for the mhd flow of casson fluid in a channel," Comput. Mater. Contin., vol. 67, no. 2, pp. 1385-1398, 2021.

[29] M. Saqib, F. Ali, I. Khan, and N. A. Sheikh, "Heat and mass transfer phenomena in the flow of Casson fluid over an infinite oscillating plate in the presence of first-order chemical reaction and slip effect," Neural Comput. Appl., vol. 30, no. 7, pp. 2159-2172, 2018.

[30] M. Das, R. Mahato, and R. Nandkeolyar, "Newtonian heating effect on unsteady hydromagnetic Casson fluid flow past a flat plate with heat and mass transfer," Alexandria Eng. J., vol. 54, no. 4, pp. 871-879, 2015. 